\title{
Cardiovascular Protection by Natural Products
}

Cardiovascular diseases are world-wide recognized leading causes of death, affecting both developed and developing countries where they represent a major economic burden. Hence, it is of great interest to look for successful treatments to increase the survival of patients suffering from these disorders and to reduce the impact of these diseases in the global health system.

Since the pathology and risk factors leading to the onset of cardiovascular diseases are complex, finding new effective drugs or new drug-targets remains a challenge to science. In fact, there has been an overall decrease of the introduction of new drugs, including those to treat cardiovascular diseases, into clinical practice during the last decades, due to poor efficacy or the presence of adverse effects. For instance, it is of major concern that since 2010, there are only three new drugs approved to treat hypertension, the most prevalent risk factor for cardiovascular morbidity and mortality.

These shortcomings in the development of new drugs are presumably due to the original singletarget design of traditional drug development programs, denoting that it is critical to recognize the multifactorial origin of diseases, especially chronic diseases, and to reshape screening strategies from one specific target to multiple targets. Therefore, this special issue is intended to bring back the attention to the need of finding new drugs capable of treating different aspects of these diseases and to the role of natural products as an essential supply of pharmaceuticals.

Nature has proven to be an unbeaten resource of unique and effective drugs, which still today represents a significant segment of the pharmaceuti- cal market. This is the case of common drugs used to treat cardiovascular diseases, such as aspirin, statins, and a number of anti-hypertensive drugs, like for instance, captopril, which has a design based on a natural product.

In addition to this, the availability of state of the art technology allowed the efficient isolation and identification of a large number of natural products during the last half a century placing at our disposal an enormous repository of natural products that should be explored as potential candidates to treat cardiovascular diseases.

For this special issue I was privileged to count on the valuable contribution of reputed scientists from different areas of research within this field to whom I am profoundly grateful. Their work provided new insights into the beneficial effects of different components present in well known plant products like olive oil, evidencing their impressive cardiovascular effects. Additionally, new pharmacological mechanisms were described for known cardioprotective plant derived compounds, findings that are in agreement with the new goal to search for different, but complementary mechanisms of action for cardiovascular drugs.

Moreover, this issue also presents supporting evidence to promote the value of the marine environment as a suitable source of products to protect the cardiovascular system, as well as discussions on novel targets to screen for new drugs and the need of data obtained from clinical studies using natural products, to better analyze the significance of in vitro experimental data.

Catherina Caballero-George

\author{
Bibliography \\ DOI http://dx.doi.org/ \\ 10.1055/s-0035-1546122 \\ Planta Med 2015; 81: 623 \\ (c) Georg Thieme Verlag KG \\ Stuttgart · New York . \\ ISSN 0032-0943 \\ Correspondence \\ Catherina Caballero-George, \\ Ph.D. \\ Group of Pharmaceutical \\ Research \\ Unit of Molecular Pharmacology \\ and Pharmacognosy \\ Institute for Scientific Research \\ and High Technology Services \\ (INDICASAT AIP) \\ Building 219, City of Knowledge \\ Panama \\ Republic of Panama \\ Phone: + 507.517.0737 \\ c.caballerogeorge@gmail.com
}

